\title{
El Principio de Inclusión: Similitudes y Diferencias en la Educación Escolar y Superior en Chile
}

\section{The Principle of Inclusion: Similarities and Differences in General and Higher Education in Chile}

\author{
Juan Pablo Queupil 1* \\ Francisco Durán del Fierro ${ }^{2}$ \\ ${ }^{1}$ Pontificia Universidad Católica de Valparaíso, Chile \\ ${ }^{2}$ Universidad de Chile, Chile
}

\begin{abstract}
Este artículo analiza la legislación vigente respecto de la inclusión educacional para la educación escolar -o enseñanza general-, y la compara con lo indicado en la propuesta de reforma a la educación superior. Así, se consideran los objetivos declarados y alcances para ambas normativas legales, complementado con un contraste entre lo existente y lo vislumbrado para dos diferentes niveles educacionales. Al revisar estas leyes, se concluye que hay diferencias sustantivas cuando se habla de inclusión en ambos niveles educacionales. En educación escolar, la inclusión se promueve en el contexto de la segregación socioeconómica de las escuelas, por tanto, se enfoca en la inclusión de estudiantes de distinto origen social y cultural al interior de ellas. En cambio, la reforma a la educación superior enfoca la inclusión desde la gratuidad y en un sistema de acceso "justo", promoviendo la inclusión de una diversidad de estudiantes en el sistema en su conjunto.
\end{abstract}

Descriptores: Educación integradora; Legislación educacional; Enseñanza general; Enseñanza superior; Chile.

This article analyzes the current legislation regarding educational inclusion for general education, and then this is contrasted with those indications about inclusion in the bill for higher education under discussion in Congress. Thus, we consider the stated objectives and scope for both legal regulations, complemented by contrasting what exists and what is envisioned for the two different levels of education. In reviewing these laws, we conclude that there are similarities and substantive differences regarding the inclusion concept in both educational levels. In fact, for general education, inclusion is promoted in the context of socioeconomic segregation of schools; therefore, it focuses on the inclusion of students of different social and cultural origin within them. On the other hand, the educational reform of higher education emphasizes inclusion from the standpoints of universal gratuity and a system of "fair" access, promoting the inclusion of a diversity of students in the system as a whole.

Keywords: Inclusive education; Educational legislation; General education; Higher education; Chile.

La investigación está enmarcada en el Proyecto PIA CONICYT CIE 16009 de la Comisión Nacional de Ciencia y Tecnología de Chile. Se agradece el apoyo del Centro de Investigación para la Educación Inclusiva, la Pontificia Universidad Católica de Valparaíso y CONICTT.

*Contacto: juan.queupil@pucv.cl

ISSN: 0718-7378

www.rinace.net/rlei/
Recibido: $\quad 13 / 11 / 2017$

$1^{a}$ Evaluación: 24/02/2018

Aceptado: 02/03/2018 


\section{Introducción}

El segundo gobierno de la Presidenta Michelle Bachelet (2014-2018) ha establecido la implementación de un programa de gobierno que reforma diferentes áreas sociales y económicas del país, incluyendo seguridad social, salud y educación, entre otros, bajo un esquema de derechos sociales.

En el sector educación se han propuesto reformas en todos los niveles, sustentadas de manera transversal en los principios de calidad e inclusión. En educación parvularia, el foco ha estado en el aumento de la cobertura para la primera infancia y la modernización de la institucionalidad pública a través de la creación de una Subsecretaría de Educación Parvularia. En la educación escolar o enseñanza general -aquella que transcurre desde $1^{\circ}$ al $12^{\circ}$ grado-, las reformas apuntaron al fin del lucro con recursos públicos y del financiamiento compartido, y de toda forma de selección de alumnos en los establecimientos educacionales, además del mejoramiento de la formación inicial a través de una nueva carrera docente y una nueva institucionalidad para la educación pública. En educación superior, en tanto, se ha propuesto introducir gradualmente la gratuidad universal, modernizar y reformar la institucionalidad pública y las políticas correspondientes ${ }^{1}$, crear dos nuevas universidades estatales en las regiones que carecían de ellas, junto con centros de formación técnica a lo largo del país (Programa de Gobierno, 2014).

A partir de estas reformas, se han discutido profunda y extensamente algunos de los principios orientadores en educación. Uno de los que ha signado este proceso ha sido el principio de inclusión, en el contex to de una creciente demanda por acceso a la educación, que algunos han denominado, al menos en el nivel terciario, como la consolidación de una masificación avanzada (Atria, 2012). Sin embargo, el debate público en educación ha sido intenso y polarizado, en particular porque la aproximación a este concepto ha estado influida por la pugna entre distintas visiones normativas² y perspectivas y políticas.

A nivel internacional, particularmente en Latinoamérica, la inclusión en educación ha estado vinculada, al menos inicialmente, a las personas en situación de discapacidad o necesidades educativas especiales, siguiendo los lineamientos indicados por la Declaración Universal de los Derechos Humanos y la Declaración Mundial sobre Educación, entre otros referentes (López-Torrijo, 2009; Muñoz, 2011), que luego se plasmaron en diversos decretos y normativas a nivel local.

Recientemente, se han ido consolidando algunos marcos teóricos, conceptuales y legales que han ampliado el campo de acción a aquellas personas que han sufrido situaciones o espacios de exclusión en el sistema educativo (Infante, 2010). A su vez, lo anterior suele asociarse con nociones y procesos de integración, diversidad, responsabilidad social y el enfoque de derechos. En este punto es importante distinguir que, por un lado, la integración se relaciona con la adaptación de las personas diferentes al sistema; por otro lado, la inclusión reconoce la diversidad, y es el sistema el que se adapta a ella (Rubio,

\footnotetext{
${ }^{1}$ Principalmente el Sistema Nacional de Aseguramiento de la Calidad, los mecanismos de financiamiento estudiantil y a las instituciones, las formas de acceso a la educación superior y los procedimientos de intervención y cierre para instituciones en crisis.

${ }^{2}$ En este caso lo normativo se entiende como el deber-ser que caracteriza a una visión de mundo. Se trata de un deber que funciona como el fundamento de cualquier acción política.
} 
2017). Es decir, son dos paradigmas diferentes que igualmente suelen usarse como sinónimos. Más allá de las distintas aproximaciones conceptuales utilizadas para hacer comprensible la noción de inclusión, es importante indagar en su uso concreto, especialmente en las normativas legales que buscan regular su aplicación en la educación, en sus diferentes niveles. En ese contex to es posible visualizar las diferencias y similitudes en juego.

En Chile existen algunas leyes que impactan directa o indirectamente en la noción de inclusión en análisis. Por ejemplo, la ley $\mathrm{N}^{\circ} 20.609$ que establece medidas contra la discriminación arbitraria ${ }^{3}$ y la ley $\mathrm{N}^{\circ} 20.422$ sobre igualdad de oportunidades e inclusión social de personas con discapacidad ${ }^{4}$. Sin embargo, en educación la noción de inclusión ha sido interpretada desde distintas aproximaciones que dependen de la posición desde la cual se enuncia su definición. Por lo tanto, la pregunta sobre cómo se expresan estas diferencias y a qué visiones normativas responden no ha sido abordada de forma precisa y suficiente.

Para este estudio, que aborda la política pública educacional en inclusión, es pertinente analizar los más recientes cuerpos legales al respecto. Según Hodder (2000), aquellos marcos legales en forma de decretos y leyes educativas podrían ser considerados una forma de producción de material cultural, dado que proponen y regulan ciertas formas de acción. A su vez, Potter y Wetherell (1987) proponen un análisis de discurso pragmático como medio a través del cual el investigador considera al discurso como acción y lo analiza por sus implicaciones pragmáticas para la vida cotidiana (Iñiguez, 2003).

Este estudio se sitúa en tal disyuntiva desde el punto de vista de las diferencias en los objetivos y alcance de la inclusión tanto en educación general como en educación superior. Para ello, se analizan dos marcos legales que, en principio, expresan visiones de inclusión diferentes que es necesario relevar para efectos de auscultar las visiones normativas que la expresan. Por una parte, se indaga en el concepto de inclusión que sustenta la ley $\mathrm{N}^{\circ}$ 20.845 y su mensaje presidencial, que regula la admisión de los y las estudiantes, elimina el financiamiento compartido y prohíbe el lucro en establecimientos educacionales que reciben fondos públicos. Por otra parte, se analiza el proyecto de ley presentado al Congreso el 4 de julio de 2016 (Mensaje $\mathrm{N}^{\mathrm{o}} 1$ 10-364) de reforma a la educación superior, y su indicación sustitutiva del 07 de abril de 2017 (Mensaje $\mathrm{N}^{\circ}$ 027-365), donde se establecen nuevos principios para el sistema, se moderniza la institucionalidad pública, se modifica el sistema de aseguramiento de la calidad, se propone un nuevo mecanismo de acceso y de financiamiento, y se fortalece el sistema estatal de instituciones de educación superior.

Para esto, el análisis se enfoca no solo en lo que se expresa explícitamente como inclusión en ambos documentos, sino también cómo se despliega este concepto a través de los diferentes componentes de las reformas expuestas. Se trata entonces de una mirada sistémica que permite interpretar las semejanzas y diferencias sustantivas más que un análisis de sus definiciones textuales. Se busca, en definitiva, relevar el sentido normativo y la visión de educación que se promueve a lo largo de todos los cambios propuestos y

\footnotetext{
${ }^{3}$ Aunque deja fuera a las instituciones de educación superior en la medida que prevalece la autonomía de estas.

${ }^{4}$ Un aspecto de esta ley que impacta en la educación superior son los ajustes que se deben realizar para que las personas en situación de discapacidad puedan rendir en igualdad de condiciones las pruebas estandarizadas que regulan el acceso a las carreras universitarias.
} 
dilucidar las diferentes nociones que se despliegan alrededor del concepto de inclusión en el sistema de educación en Chile.

Para comprender el análisis propuesto es necesario describir previamente -y de manera breve- la estructura institucional y de financiamiento de cada nivel de formación puesto que condicionan, en alguna medida, la forma que adquiere la noción de inclusión. En Chile, la educación general incluye ocho años de educación primaria (conocida como educación o enseñanza básica) y cuatro años de educación secundaria (o enseñanza media), siendo estos 12 años obligatorios de acuerdo a la ley $\mathrm{N}^{\circ} 19.876$ del año 1993. A la fecha, existen tres tipos de establecimientos educacionales: públicos o municipales, particulares subvencionados, y particulares pagados. Los primeros reciben financiamiento completamente público y dependen del nivel local por medio de las Municipalidades; los segundos reciben un financiamiento parcial del Estado a través de una subvención o voucher por alumnos matriculados junto con otros recursos parciales que suscriben las familias de los estudiantes (financiamiento compartido); y los últimos no reciben financiamiento público, y sus recursos provienen principalmente de las familias, las cuales suelen ser más acaudaladas que aquellas que atienden los dos primeros tipos de escuelas, operando el sistema completo bajo un modelo de subsidio a la demanda (Gallego y Sapelli, 2007).

Es importante mencionar que actualmente se está implementando una reforma en la cual los colegios deberán decidir si serán completamente públicos o privados, además de un nuevo sistema para la educación pública que comienza su fase piloto el año 2018, donde los colegios públicos ya no dependerán administrativa y financieramente de los Municipios, sino que de un nuevo ente estatal denominado Servicio Local de Educación Pública, creándose una nueva institucionalidad decretada en la ley $\mathrm{N}^{\circ} 21.040$.

Por otro lado, la educación superior es de carácter voluntaria para los que egresan de la educación secundaria, existiendo tres tipos de instituciones: Centros de Formación Técnica (CFT), Institutos Profesionales (IP) y Universidades. Los CFT ofrecen carreras de dos años de duración que conducen a títulos técnicos de nivel superior. Los IP pueden impartir carreras técnicas y profesionales, pero no otorgan licenciaturas. De hecho, son las Universidades las que entregan este grado académico, además de títulos profesionales, y grados de magíster y doctorado en diversas áreas y especialidades.

De acuerdo con una serie de reformas aplicadas en la década de los '80, en la educación superior se permitió la creación de instituciones privadas, mientras las instituciones públicas fueron fragmentadas en diversas sedes, introduciéndose a su vez el autofinanciamiento. Así, son los propios estudiantes o sus familias las que financian los aranceles y matrículas, aunque algunos, sobre la base del mérito académico y situación económica, puedan acceder a becas o créditos. Solo recientemente se han promovido políticas orientadas a aliviar el aspecto financiero del ingreso a la educación superior a través de la gratuidad para aquellos estudiantes pertenecientes al 60\% de las familias con menores ingresos (puede consultarse más información en www.gratuidad.cl).

\section{En búsqueda de la inclusión en el sistema escolar}

\subsection{Preludio de un cambio}

En las últimas cuatro décadas, el camino que ha tomado la educación escolar ha estado marcado por un largo y extenso proceso de privatización, permitido por la introducción 
de una estructura jurídica institucional ${ }^{5}$ que organiza a la educación como un mercado . $^{6}$ Esta base estructurante ha sido complementada por una serie de políticas y programas que han profundizado este proceso. Por una parte, la introducción de la subvención escolar por estudiante para todas las escuelas, independiente de su propiedad, y posteriormente la introducción y extensión del financiamiento compartido entre las familias y la subvención escolar; y, por otra parte, la entrega de recursos públicos para expandir las escuelas privadas -infraestructura- durante los años noventa ${ }^{7}$. Estas políticas -entre otras ${ }^{8-}$ profundizaron la privatización de la educación chilena (Bellei, 2015).

La aplicación de la triada del mercado en educación escolar -elección de escuelas, competencia y privatización- ha tenido como consecuencia la segregación socioeconómica y académica de las escuelas en su conjunto. En paralelo, la educación pública ha visto reducida su matrícula, así como también la calidad que ofrece, favoreciendo la expansión del sector privado (Riesco, 2007).

Uno de los aspectos más delicados que ha promovido la lógica de mercado en educación ha sido la exclusión social de los estudiantes, aplicada mayoritariamente por los establecimientos particulares pagados y subvencionados. La libertad de enseñanza ha sido utilizada para seleccionar a los estudiantes por distintos motivos, ya sean académicos ${ }^{9}$, económicos, religiosos e, incluso, por apariencia personal. Para ello, se han instalado masivamente mecanismos de selección de estudiantes y, por cierto, de sus familias, tanto en los procesos de admisión como durante el proceso formativo, denominada expulsión selectiva (Mensaje $\mathrm{N}^{\circ}$ 131/362).

A partir de las consecuencias producidas por organizar la educación de esta forma, expresada en la segregación social y dudosa calidad de las escuelas, evidenciado por numerosas investigaciones en los últimos años (Elacqua y Santos, 2013; Flores y Carrasco, 2013; Gallego y Hernando, 2009; Treviño, Valenzuela y Villalobos, 2014; Valenzuela, Bellei y de los Ríos, 2010 y 2014), se ha comenzado a cuestionar los logros en equidad del sistema en su conjunto. Más en específico, la introducción de los procesos de selección ha agravado la segregación aludida en la medida que restringe las experiencias educativas a espacios socialmente homogéneos. Así, la exclusión o "eliminación” de estudiantes de los proyectos educativos no consigue contemplar el principio de inclusión, entendido como la garantía de educación para todas y todos, sin discriminación arbitraria. Es decir, la segregación social en educación, producto de este proceso de mercantilización introducido desde diferentes políticas y programas, tuvo como consecuencia la instalación y demanda de mayor inclusión.

En este contexto, en 2014-con la llegada de un nuevo gobierno- se propone una Ley de Inclusión con el objetivo de resolver algunos de los aspectos más problemáticos en esta

\footnotetext{
5 La consolidación del Estado subsidiario, así como también la introducción de políticas de financiamiento y la reestructuración de la educación pública.

${ }^{6}$ En términos conceptuales y prácticos hay una diferencia sustancial entre organizar un sistema bajo la lógica del mercado y la existencia de entidades privadas que ofrecen educación. Es empíricamente viable tener un sistema de educación con entidades privadas pero que no operen bajo la lógica de mercado.

${ }^{7}$ En compensación por la extensión de la jornada escolar.

${ }^{8}$ Es necesario destacar también la descentralización de la educación pública y el aumento del voucher para los estudiantes más pobres.

${ }^{9}$ Lo que incluye las necesidades educativas especiales.
} 
materia. Luego de la correspondiente discusión parlamentaria, en 2015 se aprueba y promulga la ley $\mathrm{N}^{\circ} 20.845$, que expresa una nueva concepción sobre inclusión ${ }^{10}$.

\subsection{Inclusión en educación escolar}

Como señala el mensaje del proyecto de ley, el objetivo de la reforma es terminar con el financiamiento compartido, la selección de estudiantes y el lucro con recursos públicos (Mensaje $\mathrm{N}^{\circ}$ 131/362). Visto en forma general, es una reforma que cambia las bases estructurales sobre las cuales descansa el sistema escolar, impulsada por la necesidad de mayor inclusión, entre otros aspectos.

La promoción de la inclusión se aprecia claramente en el segundo componente de la reforma, que es la modificación del mecanismo de selección de los establecimientos escolares. La justificación de esta medida se basa en que la selección de estudiantes profundiza la segregación social; es decir, la competencia entre establecimientos incentiva que estos seleccionen a aquellos estudiantes con mejor rendimiento académico o capacidad económica, o a los que mejor se ajustan a su proyecto educativo. Adicionalmente, hace inviable la elección de los padres puesto que son los establecimientos los que finalmente deciden quienes entran. De esta forma,

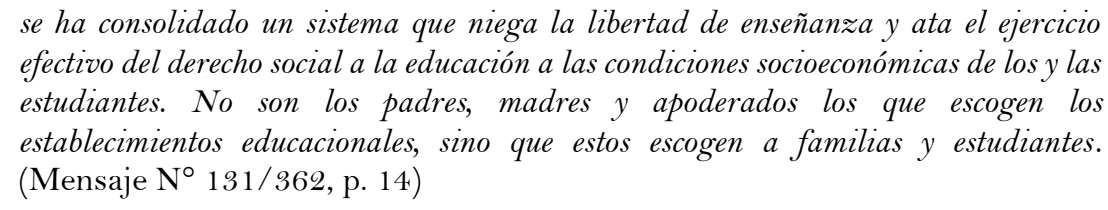

Por lo tanto, la selección de estudiantes produce discriminaciones en términos económicos y sociales que no permite un desarrollo integral de la educación. Se consolida una educación que excluye y discrimina a estudiantes y familias y, de esa forma, se reproducen de forma explícita las desigualdades sociales previas. La educación, bajo este esquema, no es sino un dispositivo que potencia las diferencias sociales y culturales, consolidando una sociedad cuyo tejido social se desvanece poco a poco.

Los fundamentos que legitimaron la reforma no solo se quedaron en eso. También se señala que un cambio en este componente redundará en mejorar la calidad del sistema educativo. Es relevante señalar esto puesto que hay un giro sustantivo en la relación entre calidad e inclusión. Si antes calidad se asociaba con identificar los mejores talentos para obtener buenos desempeños o resultados en el Sistema de Medición de la Calidad de la Educación (SIMCE) y la Prueba de Selección Universitaria (PSU) ${ }^{11}$, esta ley identifica calidad con hacerse cargo de diferentes perfiles de estudiantes. Es decir, cuestiona los hallazgos de investigaciones que indican que los mejores resultados de los establecimientos que seleccionan se explican principalmente por el perfil socioeconómico de los estudiantes y no por la efectividad de sus procesos formativos (Carrasco et al., 2014; Contreras et al., 2011; MacLeod y Urquiola, 2012). Por tales motivos, se apela a que la calidad no solo se expresa en los resultados, sino también en los procesos que se diseñan

\footnotetext{
${ }^{10}$ Es necesario tener presente que esta ley pone su foco en la inclusión pero no es la única temática que aborda. Su propio título lo deja en claro: "de inclusión escolar que regula la admisión de los y las estudiantes, elimina el financiamiento compartido y prohíbe el lucro en establecimientos educacionales que reciben aportes del estado".

${ }^{11} \mathrm{El}$ SIMCE es un conjunto de pruebas que se aplican en varios niveles de la educación escolar para medir el dominio del currículum nacional. La Prueba de Selección Universitaria (PSU) es una batería de instrumentos de evaluación que se aplica para seleccionar a los estudiantes que postulan a las universidades adscritas al Sistema Único de Admisión (SUA).
} 
y aplican para mejorar la enseñanza y aprendizaje a un conjunto más amplio y diverso de estudiantes.

En resumen, las razones esgrimidas para impulsar el fin de la selección fueron tres: (i) mejorar la calidad del sistema; (ii) garantizar la libertad de elección de las familias; y (iii) disminuir la segregación escolar (Mensaje $\mathrm{N}^{\circ}$ 131/362). En este sentido, la eliminación de la selección busca mejorar la calidad del sistema educativo y construir una sociedad más integrada y cohesionada (Mensaje $\mathrm{N}^{\circ}$ 131/362). Como se aprecia, la noción de inclusión que se promueve pone énfasis en la integración social, es decir, en la necesidad de conformar espacios educativos socialmente heterogéneos.

Los procesos formativos en estos espacios favorecen la apropiación de valores democráticos, generosidad, responsabilidad, respeto, valoración por el otro e igualdad (Rao, 2013). Asimismo, la construcción de estos espacios tendría impacto positivo en la formación de los estudiantes en la medida que aumenta la motivación y rendimiento de aquellos de menor nivel socioeconómico, sin afectar el desempeño del resto de los estudiantes (Bellei, 2013; Kahlenberg, 2012). Es decir, es una inclusión que no solo apela al acceso sin discriminaciones, sino también a consolidar la progresión formativa y la participación en la comunidad escolar.

Con ello, se piensa en salas de clase como espacios inclusivos donde conviven estudiantes con diferente capital cultural, económico y social. La experiencia educativa en estos espacios tendría efectos positivos para la sociedad por cuanto se cruzan distintas miradas, visiones, motivaciones y expectativas de vida que se enriquecen y complementan. El convencimiento del valor de la integración social en los procesos educativos es la base de la Ley de Inclusión.

De hecho, en el articulado de la ley queda claramente establecido cuando se introducen las modificaciones al decreto con fuerza de ley $\mathrm{N}^{\circ} \mathcal{Q}$ de la ley $\mathrm{N}^{\circ}$ 20.370. Se señala en el artículo 3 letra $K)$ :

Integración e inclusión. El sistema propenderá a eliminar todas las formas de discriminación arbitraria que impidan el aprendizaje y la participación de los y las estudiantes. Asimismo, el sistema propiciará que los establecimientos educativos sean un lugar de encuentro entre los y las estudiantes de distintas condiciones socioeconómicas, culturales, étnicas, de género, de nacionalidad o de religión. (Ley $\mathrm{N}^{\circ}$ $20.845,2015$ p. 2)

La ley no solo establece la ilegalidad de la aplicación de mecanismos de selección en los establecimientos escolares a través de lo que llama discriminación arbitraria, sino también introduce la obligación al sistema en su conjunto de propiciar espacios educativos heterogéneos. Es decir, introduce una obligación jurídica positiva que establece que el sistema tiene que perseguir principios que se asumen como justos. Entre ellos se encuentran los valores del pluralismo, la tolerancia y respeto por el otro, y los valores cívicos y democráticos. No se trata simplemente de asegurar un derecho de no interferencia -la no discriminación-, los que generalmente se vinculan con la libertad de enseñanza, sino de comprometer una correspondencia de principios entre el sistema y las diferentes partes -las escuelas en este caso-que lo componen.

Por lo tanto, el proyecto se hace cargo del problema de la segregación socioeconómica del sistema e impulsa una mayor inclusión por medio dela integración y cohesión social. El medio para hacer viable esta integración social es la eliminación de cualquier tipo de selección. Es decir, es una inclusión entendida esencialmente como no selección cuyo fin 
es la integración social al interior de las escuelas. En definitiva, se trata de una inclusión que no solo rompe con la selección sino también que promueve acciones orientadas a cumplir un determinado fin.

\section{La visión de inclusión para la educación superior}

\subsection{La propuesta que plantea la reforma}

La reforma educacional en marcha pretende avanzar en una educación que instale las bases para un país más justo e inclusivo. El hecho que la reforma destaque lo relacionado con inclusión, ya sea semánticamente o como objetivo general de sociedad, motiva su examen y análisis. Así, en cuanto a la educación superior, el gobierno envía al congreso el mensaje 110/364 del 4 de julio de 2016, con el cual se da inicio al proyecto de reforma de la educación superior en Chile.

El proyecto se plantea desde tres ejes: calidad, pertinencia y equidad e inclusión. Estos principios se consideran como las bases para el sistema educativo terciario. Lo primero que llama la atención es que equidad e inclusión se consideran desde un punto de vista amplio. Así, para esta reforma, tanto la equidad como la inclusión deberían considerar que,

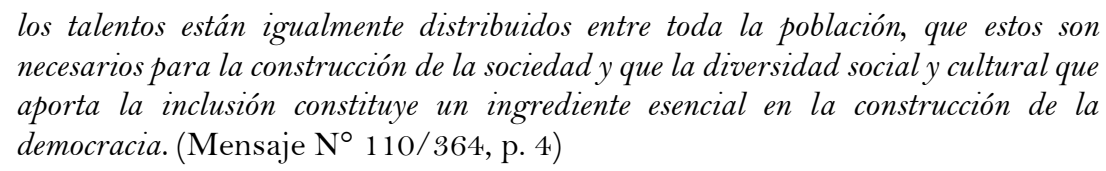

En esta declaración destaca la asociación de la inclusión con aspectos relacionados con la diversidad de talentos que hay de forma transversal en la sociedad, bajo la lógica implícita que más inclusión implica más diversidad de estudiantes en el sistema. En ese sentido,

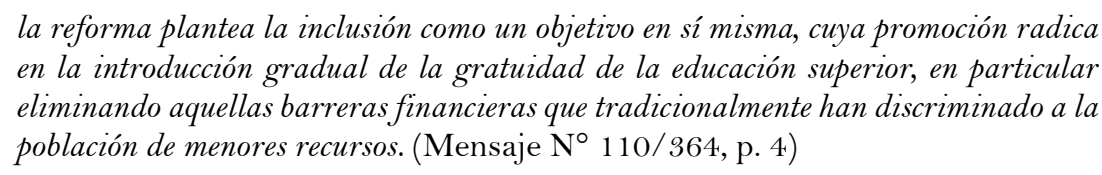

Por otro lado, el proyecto de ley original es cauto en mantener la pluralidad de proyectos y misiones de las instituciones de educación superior. Esto puede sonar contradictorio, pero a la vez sensato. En Chile, y en lo que parece ser una realidad a nivel mundial, las instituciones de educación superior gozan de plena autonomía académica, financiera y administrativa. Sin embargo, esta misma autonomía ha estado expuesta a críticas respecto del límite que le da sentido, o más específicamente, de lo poco inclusivas que podrían ser algunas instituciones, ya sea por razones de credo, dogma, o reglamento institucional (Fernández, 2013; León, 2011). Esto podría ser contraproducente para los aspectos de equidad y participación que aboga la inclusión.

Para la educación superior, en pos de promover la equidad y la inclusión, el proyecto de ley original propone dos medidas específicas: 1) financiamiento gratuito de la educación superior, y 2) desarrollo de un sistema de acceso.

Respecto del financiamiento, se busca aliviar el gasto en que incurren las familias para que sus hijos ingresen a la educación post-secundaria. Teniendo en cuenta que el gasto privado es uno de los más altos entre los países de la OCDE (OCDE, 2015), alcanzar la gratuidad de forma paulatina parece una medida razonable y adecuada. Además, se incorporan exigencias normativas (p. ej. prohibición taxativa del lucro) y de calidad para las 
instituciones que desean suscribirse a esta medida, lo cual implica, al menos como discurso, la promoción de una inclusión responsable.

En cuanto a la segunda línea de acción, se indica de forma laxa que "se debe mejorar la forma en que los estudiantes acceden a la educación superior" para así lograr mayor equidad e inclusión (Mensaje $\mathrm{N}^{\circ} 110 / 364$, p.23). Eso sí, se declaran cuatro objetivos específicos para aquello: 1) información transparente y clara de la oferta académica, disponible para todos los estudiantes por igual, 2) procesos de acceso justo, 3) respeto por las preferencias de estudiantes y autonomía de las instituciones y sus carreras, y 4) orientación hacia la calidad y desarrollo del país a través de diversas políticas públicas. Como puede notarse, la equidad e inclusión se asocian a aspectos tangenciales de la igualdad y justicia, y se enfocan más bien en aspectos como la calidad, autonomía y transparencia, es decir, elementos transversales que últimamente se promueven con fuerza en el sistema de educación superior.

De hecho, los aspectos indicados son principios que orientan la reforma, en línea con la ley general de educación, conocida como LGE (Ley $\mathrm{N}^{\circ} 20.370$, 2009). En particular, la reforma indica explícitamente que el sistema de educación superior -donde se reconocen el subsistema universitario y el técnico profesional- "debe promover la inclusión de las y los estudiantes en las instituciones de educación superior, velando por la eliminación de todas las formas de discriminación arbitraria”. Acá es interesante notar que se contempla toda forma de discriminación, más allá de las financieras, siendo estas últimas abarcadas por las medidas asociadas con gratuidad. Por ende, este apartado también considera el respeto hacia la diversidad de culturas, orientaciones sexuales, identidades de género, situación de discapacidad y orígenes socioeconómicos.

\subsection{El rol de una nueva estructura legal para la inclusión}

En cuanto a la estructura institucional de la reforma, la propuesta de ley considera la creación de una Subsecretaría de Educación Superior, la cual tendrá dentro de sus funciones y atribuciones el proponer al Ministerio de Educación políticas de acceso e inclusión. A su vez, y retomando la medida relacionada con el desarrollo de un sistema de acceso, la reforma plantea que este sea administrado por la Subsecretaría, tomando en consideración, entre otras leyes, la ley $\mathrm{N}^{\circ} 20.422$, la cual establece normas sobre igualdad de oportunidades e inclusión social de personas con discapacidad.

Por otro lado, la reforma establece un Sistema Nacional de Aseguramiento de la Calidad de la Educación Superior, al cual le corresponderá el desarrollo de políticas que promuevan la calidad, pertinencia y articulación de las instituciones de educación superior, pero también su inclusión y equidad.

Además, el proyecto de ley hace especial hincapié en las instituciones de educación superior estatales, las cuales deben guiarse por los principios que define la reforma, en particular, el desarrollo integral, sostenible e inclusivo del país, y serán responsables de la igualdad de oportunidades de sus estudiantes (entendida por la reforma como equidad) y de la aplicación de políticas de acceso e inclusión que disponga la Subsecretaría.

Es importante destacar que nueve meses después del ingreso del proyecto de ley, el gobierno envía una indicación sustitutiva fruto del debate nacional y el proceso de reflexión ocurrido en el propio Congreso. Si bien se reafirman los aspectos relacionados con la inclusión y equidad del proyecto original -vale decir, la instauración de la gratuidad y un nuevo sistema de acceso para la educación superior-, no se entregan mayores 
antecedentes al respecto. Así, en cuanto a la inclusión, sigue presente en los distintos componentes de la reforma y, además, como principio que inspira el devenir del sistema en su conjunto.

\section{Contrastes en inclusión en el sistema de educación chileno}

En el sistema educacional chileno, la inclusión -y por extensión la equidad educacional-, ha evolucionado de forma gradual e incluso tardía. Si bien el concepto es manejado en las esferas de la política pública y en las propias instituciones de educación, con distintas interpretaciones, su ámbito legal ha quedado formalmente establecido recientemente en la última década. Así, por ejemplo, la ley $\mathrm{N}^{\circ}$ 20.422, la cual establece normas sobre igualdad de oportunidades e inclusión social de personas con discapacidad, fue promulgada recién el 10 de febrero de 2010. Asimismo, la ley $\mathrm{N}^{\circ} 20.609$ que establece medidas contra la discriminación se promulgó el 12 de julio de 2012.

$\mathrm{Al}$ analizar ambos proyectos de ley, cabe preguntarse si las políticas de inclusión para ambos ciclos de educación son similares o coherentes entre sí. Una respuesta preliminar en base a la revisión de este estudio indicaría que hay diferencias y matices al respecto, pero también algunas semejanzas.

En relación con la educación escolar, como se ha mencionado, el año 2015 se aprobó la ley $\mathrm{N}^{\circ} 20.845$ de Inclusión Escolar, que regula la admisión de los y las estudiantes, elimina el financiamiento compartido y prohíbe el lucro en establecimientos educacionales que reciben aportes del Estado. En esta ley queda efectivamente establecida la noción de inclusión que regirá al sistema escolar. Se trata de una inclusión asociada a la integración y cohesión social al interior de los establecimientos escolares: propiciar la diversidad social, económica y cultural interna de las escuelas. Esta promoción expresa, a su vez, la valoración de la sociedad como una comunidad política que se preserva a través del desarrollo de espacios socialmente inclusivos. La escuela, se asume, no puede operar como un mecanismo de diferenciación o distinción social; al contrario, es el lugar donde distintas visiones y experiencias deben reunirse, comprenderse y desarrollarse con miras a la integración social.

Cabe preguntarse, sin embargo, la motivación que sustenta esta idea de inclusión en educación escolar. De lo analizado se puede inferir que la noción promovida depende de dos elementos. Por una parte, del diagnóstico que la segregación social de las escuelas trastoca las bases sobre las cuales se legitiman las sociedades democráticas. El convencimiento de que efectivamente la segregación es un problema, es parte fundamental de la promoción de este tipo de inclusión. Esto no es trivial puesto que el consenso de que un problema es un problema no es un asunto meramente procedimental, es más bien una discusión que moviliza elementos sustantivos. Esto último conduce al segundo punto en cuestión.

Efectivamente, considerar este fenómeno como un problema es asumir, a la vez, una noción particular de justicia social. No solo se trata de asegurar la igualdad de oportunidades, es decir, ofrecer a todos la posibilidad de una educación en base al mérito, donde la distribución posterior en la jerarquía de ingresos o posición social depende del esfuerzo, a pesar de que hay bastante de eso también. Se trata de un giro hacia una suerte de igualdad de posiciones que busca hacer más próximas las experiencias educativas. No 
obstante, esta noción de igualdad no implica necesariamente reducir las desigualdades o buscar la igualdad completa en las sociedades, sino de fijar ciertas posiciones de proximidad para legitimar los resultados posteriores, aunque sean desiguales (Dubet, 2014). Dicho en otros términos, la heterogeneidad social al interior de las escuelas es un valor y una necesidad en la medida que legitima la desigualdad posterior (Marshall, 1965). El punto relevante es que la noción de inclusión promovida expresa un giro en la idea de justicia social hasta ese momento utilizada: ya no solo es necesario asegurar la igualdad de oportunidades -acceso en base al mérito- sino también una cierta igualdad de posiciones con la finalidad de reducir las distancias en la estructura social. La forma para darle sentido a ese propósito de igualdad es que todos los miembros de una sociedad tengan el derecho a una educación que promueva ciertos valores compartidos ${ }^{12}$.

Por su parte, el proyecto de ley de reforma a la educación superior, en sus distintas versiones, establece la inclusión como principio orientador del sistema; sin embargo, no se especifica su contenido y sentido en las normas regulatorias que se proponen. Es decir, se postula el principio de inclusión pero no se visualiza cómo su contenido se desplegará en el sistema en su conjunto, tanto en las atribuciones de los organismos públicos como en las obligaciones de las instituciones de educación superior.

A pesar de estas dificultades prácticas, conceptualmente inclusión se asocia a la ampliación de la igualdad de oportunidades a través de la identificación de talentos en sus distintos contextos. Se trata de resguardar y promover una diversidad social, económica y cultural en el sistema en su conjunto. No se observa, por tanto, una preocupación por constituir espacios o experiencias formativas entre distintas posiciones sociales. Lo esencial es que el sistema de educación superior acoja a la diversidad de estudiantes que pretenden continuar estudios superiores, independientemente de si se conforman espacios sociales homogéneos entre instituciones y si algunas instituciones son más selectivas que otras.

Por lo tanto, en educación superior la noción de justicia social, que se deriva del principio de inclusión propuesto, se asocia más bien a la ampliación de oportunidades de acceso. Esto implica que el objeto de la inclusión es que los estudiantes no tengan trabas económicas para ingresar y que el mérito sea el principal factor para obtener una vacante. El mérito, en este caso, se presenta como la forma que permite que dicha distribución no esté influenciada por factores ajenos al desempeño académico, como pudieran ser algunos privilegios sociales.

La idea de que los talentos se encuentran igualmente distribuidos entre ricos y pobres requiere de la promoción de esta idea. Con ello, en educación superior lo que prevalece es la preocupación por asegurar las oportunidades de acceso a una población cada vez más heterogénea de estudiantes, valorando sus respectivas trayectorias formativas, así como también sus competencias y habilidades. Por tanto, en este caso no se trata de reconfigurar los espacios sociales de formación al interior de las instituciones de educación superior, sino de refinar los mecanismos de acceso a fin de que reconozcan a la heterogeneidad de estudiantes y, de ese modo, asegurar la igualdad de oportunidades.

Ciertamente, es una diferencia sustantiva la que se observa en ambos niveles educativos. En educación escolar se induce la diversidad en los propios establecimientos escolares; en

${ }^{12}$ Esto giro es, por cierto, limitado, puesto que la reforma no consideró a los establecimientos privados, lo que limita el alcance de esta idea de "igualdad" al dejar intacta la jerarquía social que produce su existencia. 
cambio, en educación superior se promueve en el sistema completo, sin especificar las instituciones. Una explicación de esta diferencia, al menos en el grado de intervención que regulan estas leyes, es la pretensión de autonomía que postulan las instituciones de educación superior. Sin embargo, coinciden en un punto: en ambos casos se utiliza un medio -integración social o mérito- para justificar la desigualdad en términos de la legitimidad que la contiene. Es decir, se diferencian en cómo se produce la desigualdad pero no cuestionan el hecho de que se produzca la desigualdad posterior, entendida como la distribución jerárquica de posiciones sociales. Por otra parte, en términos específicos, se identifican al menos tres formas de entender la inclusión en educación, a partir de la revisión de las respectivas leyes y la experiencia práctica del sistema educacional chileno para ambos niveles: como no discriminación, no selección y selección por mérito académico.

La primera hace referencia a la eliminación de cualquier tipo de discriminación arbitraria, ya sea económica, territorial, de género o por situación de discapacidad. La segunda supone la no selección por ningún tipo de condición, económica o académica. La tercera supone la introducción de mecanismos selectivos que permitan identificar los talentos académicos independiente del contexto económico y social. La primera y segunda es inclusión como integración social; la tercera es inclusión como excelencia o talento académico. La primera es promovida tanto por la educación escolar como por la educación superior. La segunda es válida para la educación escolar, y la tercera para la educación superior ${ }^{13}$.

Llegado a este punto, es importante señalar una particularidad en el caso de la educación superior que permite entender la propuesta de ley en análisis ${ }^{14}$. Una parte del sistema, exclusivamente universidades, participa de procesos de admisión basados en la Prueba de Selección Universitaria (PSU). Es decir, considera que la inclusión se sostiene en el mérito académico, que se expresa en los resultados de estas pruebas. Por otra parte, hay instituciones de educación superior -universidades, IP y CFT- que no consideran los resultados de esta prueba, e incluso no utilizan ningún mecanismo de selección más que el orden de llegada. Esto muestra una diferencia sustantiva en la forma en que se entiende la inclusión en educación superior. Algunas la asimilan al mérito mientras que otras la conciben como acceso abierto sin ninguna restricción más que el pago de un arancel ${ }^{15}$. En definitiva, actualmente en educación superior hay una escisión en términos de lo que se comprende la inclusión que tiene consecuencias en el sistema en su conjunto (cuadro 1).

En definitiva, la inclusión en educación es resultado de una serie de relaciones de poder que expresan una visión de sociedad. En educación escolar (de $1^{\circ}$ a $12^{\circ}$ grado) se produjo un cambio en las relaciones que ejercían influencia es su contenido. Se transitó desde una inclusión entendida como igualdad de oportunidades a una que fija ciertas condiciones de posición y proximidad social con miras a legitimar los resultados sociales que se

\footnotetext{
${ }^{13}$ Como se mencionó anteriormente, la educación superior, a diferencia de la educación escolar, no es obligatoria en términos legales, cuestión que en alguna medida afecta en la noción de inclusión. Sin embargo, esta relación no es totalmente directa puesto que a nivel internacional es posible encontrar experiencias donde la educación superior es no selectiva, en todos sus niveles (técnico, profesional, etc.).

${ }^{14}$ La propuesta de reforma a la educación superior expresa esta situación paradójica - dos formas de entender la inclusióny de alguna manera no logra zanjarla.

${ }^{15}$ De cierta forma, se podría argüir que las instituciones de educación superior que no exigen ningún requisito de ingreso apelan a una idea de inclusión parecida a la que establece la Ley de Inclusión en educación escolar, sin embargo, la diferencia radica en que estas instituciones segregan socialmente, cuestión que es precisamente lo que quiere reducir la ley en educación escolar.
} 
produzcan. En educación superior, por su parte, si bien prevalece la igualdad de oportunidades, la pugna de visiones ha fragmentado su contenido, y la propuesta de reforma no resuelve del todo esta tensión. Es decir, la igualdad de oportunidades es hegemónica pero se bifurca en dos interpretaciones: por una parte, inclusión con excelencia, esto es, ampliar las oportunidades de acceso pero siempre manteniendo un umbral -de resultados-, el que se puede verificar por medio de múltiples instrumentos; y, por otra parte, inclusión como no selección, entendida como acceso sin condiciones académicas.

Cuadro 1. Resumen de resultados del análisis

\begin{tabular}{|c|c|c|c|}
\hline NiVEL & $\begin{array}{l}\text { OBJETIVOS DE LA } \\
\text { NORMATIVA }\end{array}$ & $\begin{array}{l}\text { DIFERENCIAS NOCIÓN } \\
\text { INCLUSIÓN }\end{array}$ & $\begin{array}{l}\text { SIMILITUDES } \\
\text { NOCIÓN } \\
\text { INCLUSIÓN }\end{array}$ \\
\hline $\begin{array}{l}\text { Educación } \\
\text { escolar }\end{array}$ & $\begin{array}{l}\text { Calidad enseñanza } \\
\text { Elección de padres } \\
\text { Sin segregación social }\end{array}$ & $\begin{array}{l}\text { Integración social } \\
\text { Espacios educativos } \\
\text { internamente heterogéneos }\end{array}$ & \multirow{2}{*}{$\begin{array}{l}\text { No discriminación } \\
\text { No selección (en } \\
\text { las IES que no } \\
\text { exigen requisitos } \\
\text { de entrada) }\end{array}$} \\
\hline $\begin{array}{l}\text { Educación } \\
\text { superior }\end{array}$ & $\begin{array}{l}\text { Equidad en el acceso } \\
\text { Calidad formativa } \\
\text { Pertinencia }\end{array}$ & $\begin{array}{l}\text { Ampliación oportunidades de } \\
\text { acceso basadas en el mérito } \\
\text { Sistema con estudiantes } \\
\text { heterogéneos }\end{array}$ & \\
\hline
\end{tabular}

Fuente: Elaboración propia.

Como se aprecia, hay diferencias formales -identificadas en el análisis de los dos proyectos de ley- en la noción de inclusión usada en educación escolar y superior. Además, si se observa el devenir del sistema en su conjunto, es posible identificar usos que muchas veces son contradictorios al interior del mismo nivel de educación. De este modo, la diversidad de interpretaciones es particularmente preocupante para las políticas educativas que tienen que orientar el desarrollo del sector.

\section{Conclusiones}

El presente estudio aborda las políticas de inclusión en la educación chilena para la educación escolar (de $1^{\circ}$ a $12^{\circ}$ grado) y la educación superior. Para ello, se considera una comparación desde la legislación existente para la primera, junto a una perspectiva de futuro para la segunda, pues para esta última se revisa la propuesta de ley que ha estado en discusión en el congreso chileno en los últimos dos años. Así, se analizan las particularidades de las políticas de inclusión desde los marcos legales para ambos niveles del sistema educativo, considerando sus objetivos y alcances.

Lo primero que es relevante destacar es que la inclusión ha sido considerada principalmente desde dos puntos de vista: el financiamiento y el acceso. Lo anterior con el propósito de revertir ciertas problemáticas sociales que han imperado en Chile en las últimas décadas, como la segregación social, económica y cultural. Por tanto, la necesidad de inclusión nace a propósito de su opuesto, esto es, la exclusión social.

En ambos niveles educativos, el financiamiento y el acceso son variables que explican el grado de inclusión que registran. En educación escolar, se espera terminar con el financiamiento compartido y con la selección de estudiantes a fin de disminuir la segregación escolar. En educación superior, ocurre algo similar con el financiamiento, sin embargo, en el acceso la situación es diferente. El objetivo es reducir la carga financiera 
pero a la vez regular las vacantes ofrecidas a través de procesos selectivos más pertinentes, al menos en las instituciones de educación superior que reciben recursos públicos.

En este sentido, si bien la reforma educacional ha intentado desmercantilizar la educación chilena, al menos en el discurso inicial, tanto las instituciones públicas como privadas siguen insertas en un sistema que mantiene mecanismos de mercado para la distribución de recursos, lo que tensiona la idea de inclusión, independiente de cómo se entienda. En el ámbito escolar, las escuelas siguen recibiendo subsidios fiscales en base al número de estudiantes que reciben y en educación superior la gratuidad se calcula en función de la cantidad de estudiantes del $60 \%$ de menores recursos que las instituciones logran matricular.

Es decir, al mantener el financiamiento a la demanda -entrega de recursos a las instituciones en función del número de estudiantes que reciben- el fomento de la inclusión pareciera conllevar una arista meramente presupuestaria, por cuanto entre más estudiantes admite una institución más financiamiento recibe. Así, en la práctica, este enfoque de la inclusión resulta limitado puesto que no solo se transforma en instrumental, sino también en el pilar a través del cual se hace sustentable una institución, perdiendo su sentido original, que incluye el acceso sin discriminación, el reconocimiento de la diversidad y la integración y cohesión social, entre otros elementos.

En el caso de la educación escolar se espera que la implementación de la Ley de Inclusión permita la efectiva elección de escuelas por parte de las familias. Si bien no hay claridad sobre la cantidad de establecimientos que pasarán a ser particulares pagados ${ }^{16}$, se estima que ello no afectará este objetivo. A partir de lo anterior se busca reducir la segregación social entre las escuelas, generando espacios educativos socialmente más heterogéneos. Ciertamente este es el objetivo más desafiante puesto que se enfrenta a patrones culturales históricamente constituidos que luchan por mantener las posiciones sociales. Además, esta ley no establece medidas para los establecimientos particulares pagados, cuestión que tensiona la idea de inclusión como integración social al dejar al margen a las clases sociales más pudientes.

Por otro lado, en educación superior se estima que los efectos de la implementación serán escalonados y dependerán de las cohortes de ingreso involucradas. Si bien el presente análisis enfatiza un enfoque de inclusión focalizado en la eliminación de barreras de acceso, no es claro cómo se abordarán los potenciales problemas relacionados con la progresión formativa y la participación de los estudiantes en sus respectivas carreras, pues la ley especifica que "son las propias instituciones las que deberán revisar y aplicar sus políticas al respecto, vinculándola a las dimensiones de docencia y resultados del proceso de formación" (Mensaje 110/364, p. 59).

De acuerdo con lo anterior, los aspectos relacionados con la inclusión, equidad e igualdad de oportunidades podrían mermarse si las cohortes de entrada y egreso son fuertemente disímiles, o si se desatiende el avance por carrera, año o curso de los estudiantes con características específicas históricamente analizadas, tales como el nivel socioeconómico, sexo, o etnia, entre otras, pudiendo incluso aparecer nuevas barreras escasamente

${ }^{16}$ La Ley permitía que los establecimientos que no quisieran regirse por la nueva normativa -sistema de acceso y fin del lucro principalmente- se convirtieran en particulares pagados. 
abordadas, como la alimentación, transporte y salud de los estudiantes en educación superior, por mencionar algunas.

Por lo tanto, en Chile, al hablar de inclusión también se desliza implícitamente la diferenciación, pues se realiza la distinción entre alguien que debe ser incluido y alguien que lo tiene que incluir. Esta paradoja puede verse como una espada de doble filo, pues cómo se entienda a la inclusión en educación puede tanto mermar la equidad e integración como apoyar inconscientemente a la segregación. Así, pueden existir prácticas discursivas, como las propias leyes analizadas en este estudio, que tengan una interpretación que desfavorezcan a la inclusión en sí, lo cual se sustenta en una potencial brecha entre el ideal (la ley) y su implementación (vale decir, su materialización).

Para Quiñones y Superveille (2009), lo anterior puede explicarse porque, en Latinoamérica, las políticas neoliberales se han insertado en un contexto de transformaciones sociales con la condición de asumir importantes segmentos de la población en situación de marginación. Así, por ejemplo, ha existido un aumento en la población migrante en Chile en los últimos años, incluyendo niños y niñas que van a diversas instituciones educacionales del país. Esto ha generado un debate no menor respecto de cómo deberían ser considerados tanto en el sistema social como educativo, repercutiendo también en aspectos culturales, empleo, salud e incluso idiomáticos, pues varios migrantes no hablan español como primera lengua, generando en algunos casos situaciones de marginación.

Dentro de los futuros estudios, cabe hacerse la pregunta si es pertinente que las políticas de inclusión para ambos ciclos sean similares o al menos en la misma línea normativa. Otros estudios deberían analizar cómo efectivamente se ha implementado la ley, en particular, cómo ha sido interpretada por los diferentes actores educacionales involucrados, sobre todo en la educación superior, ya sea a escala local, regional o nacional. Este análisis permitiría evaluar las implicancias reales en la inclusión de estas y otras normativas, lo cual es una evidencia necesaria, en virtud de los cambios y desarrollo a los que aspira lograr el país. Este análisis también debiera incluir las otras variables que la afectan, como el financiamiento y la calidad.

Por otro lado, en Chile se han creado dos centros de investigación con el apoyo del sector público para investigar específicamente aspectos relacionados con justicia educacional y educación inclusiva (CONICYT, 2016). Esto muestra la importancia que tendrá estos temas durante los años venideros, donde se vislumbra una mayor cantidad de líneas de investigación en esta materia.

Este ejercicio de análisis sobre un principio orientador en un sector tan relevante como es educación muestra la necesidad de su definición, en términos sustantivos. No parece plausible coordinar un sistema público sin tener antes la claridad sobre cómo las instituciones que lo conforman van a contribuir a su construcción. Esta reflexión permite aclarar ciertos aspectos que hasta ahora han sido confusos, no solo por las diferentes formas de abordar la inclusión en educación en los marcos legales propuestos, sino también por el uso retórico que se hace de ella, que muchas veces introduce nuevas ambigüedades. 


\section{Referencias}

Atria, R. (2011). Chile: La débil distinción público/privado en un sistema de educación superior de modernización avanzada. En J. J. Brunner y C. Peña (Eds.), El conflicto de las universidades: Entre lo público y lo privado (pp. 161-186). Santiago de Chile: Ediciones Universidad Diego Portales.

Bellei, C. (2013). Equidad educativa y dinámicas de mercado en educación. En Cátedra Michel Foucault de la Universidad de Chile. e Institut Français de Chile. (Eds.), Actas VI Escuela de la Cátedra Michel Foucault (pp. 47-59). Santiago de Chile: Universidad de Chile.

Bellei, C. (2015). El gran experimento: Mercado y privatización de la educación chilena. Santiago de Chile: LOM Ediciones.

Carrasco, A., Bogolasky, F., Flores, C., Gutiérrez, G. y San Martin, E. (2014). Selección de estudiantes y desigualdad educacional en Chile: ¿Qué tan coactiva es la regulación que la prohíbe? Santiago de Chile: Ministerio de Educación.

Contreras, D., Sepúlveda, P. y Bustos, S. (2011). When schools are the ones that choose: The effects of screening in Chile. Social Science Quarterly, 91(5), 1349-1368.

Dubet, F. (2014). Repensar la justicia social: Contra el mito de la igualdad de oportunidades. Buenos Aires: Siglo XXI Editores.

Elacqua, G. y Santos, H. (2013). Los efectos de la elección escolar en la segregación socioeconómica en Chile: Un análisis georreferenciado. Recuperado de http://www.espaciopublico.cl

Fernández, E. (2013). La relación entre el estado y las instituciones de educación superior. Santiago de Chile: Aequalis.

Flores, C. y Carrasco, A. (2013). (Des)igualdad de oportunidades para elegir escuela. Preferencias, libertad de elección y segregación escolar. Recuperado de http://espaciopublico.cl

Gallego, F. y Hernando, A. (2009). School choice in Chile: Looking at the demand side. Santiago de Chile: Pontificia Universidad Católica de Chile.

Gallego, F. y Sapelli, C. (2007). El financiamiento de la educación en Chile: Una evaluación. Revista Pensamiento Educativo, 4O(1), 263-284.

Hodder, I. (2000). The interpretation of documents and material culture. En N. Denzin y Y. Lincoln (Eds.), Handbook of qualitative research (pp. 393-402). Thousand Oaks, CA: Sage.

Infante, M. (2010). Desafíos a la formación docente: Inclusión educativa. Estudios Pedagógicos, 36(1), 287-297. https://doi.org/10.4067/So718-07052010000100016

Iñiguez, L. (2003). Análisis del discurso: Manual para las ciencias sociales. Barcelona: UOC.

Kahlenberg, R. (2012). From all walks of life. New hope for school integration. American Educator Review, 4, 201-235.

León, J. L. (2011). El conflicto de las universidades desde una perspectiva jurídica: lo público y lo privado en el debate sobre la autonomía universitaria. En J. J. Brunner y C. Peña (Eds.), El conflicto de las universidades: Entre lo público y lo privado (pp. 299-330). Santiago de Chile: Ediciones Universidad Diego Portales.

López-Torrijo, M. (2009). La inclusión educativa de alumnos con discapacidades graves y permanentes en la Unión Europea. RELIEVE. Revista Electrónica de Investigación y Evaluación Educativa, 15(1), 1-20. 
MacLeod, W. B. y Urquiola M. (2012). Anti-lemons: School reputation and educational quality. Recuperado de https://cloudfront.escholarship.org/dist/prd/content/qt3rc708kd/qt3rc708kd.pdf

Marshall, T. (1965). Class, citizenship and social development. Nueva York, NY: Anchor Books.

Mensaje $N^{\circ}$ 131/362. (2014). Cámara de diputados de la República de Chile. Santiago de Chile.

Mensaje $N^{\circ}$ 110-364. (2016). Mensaje de S.E. la Presidenta de La Republica con el que inicia un proyecto de ley de educación superior. Santiago de Chile.

Mensaje No 027-365. (2017). Formula indicación sustitutiva al proyecto de ley de educación superior. Santiago de Chile.

Muñoz, A. P. (2011). Inclusión educativa de personas con discapacidad. Revista Colombiana de Psiquiatría, 4O(4), 670-699. https://doi.org/10.1016/So034-7450(14)60157-8

OCDE. (2015). Education at a glance 2015. París: OCDE.

Potter, J. y Wetherell, M. (1987). Discourse and social psychology: Beyond attitudes and behaviour. Londres: Sage.

Quiñones, M. y Supervielle, M. (2009). ¿Es posible concebir políticas de inclusión social alternativas? Psicoperspectivas, 8(2), 57-81.

Riesco, M. (2007). Se derrumba un mito: Chile reforma sus sistemas privatizados de educación y previsión. Santiago de Chile: Centro de Estudios Nacionales de Desarrollo Alternativo.

Rubio, V. (2017). Inclusión de personas en situación de discapacidad en educación superior, desde el enfoque de la responsabilidad social, en un contexto de transiciones discursivas respecto del binomio integración/inclusión. Revista Latinoamericana de Inclusión Educativa, 11(2), 199-2 16. https://doi.org/10.4067/S0718-73782017000200013

Treviño, E., Valenzuela, J. P. y Villalobos, C. (2014). ¿Se agrupa o segrega al interior de los establecimientos escolares chilenos? Santiago de Chile: Universidad de Chile.

Valenzuela, J. P., Bellei, C. y De Los Ríos, D. (2010). Segregación escolar en Chile. En S. Martinic y G. Elacqua (Eds.), ¿̇Fin de ciclo? cambios en la gobernanza del sistema educativo (pp. 209-229). Santiago de Chile: Universidad de Chile.

Valenzuela, J. P., Bellei, C. y De Los Ríos, D. (2014). Socioeconomic school segregation in a marketoriented educational system. The case of Chile. Journal of Education Policy, 29(2), 217-241. https://doi.org/10.1080/02680939.2013.806995

\section{Breve CV de los autores}

\section{Juan Pablo Queupil}

Ingeniero Civil Industrial y Magíster en Gestión y Dirección de Empresas (MBA) de la Universidad de Chile. Doctor en Educación de la Universidad Estatal de Pennsylvania, Estados Unidos. Actualmente es investigador del Centro de Investigación para la Educación Inclusiva, de la Pontifica Universidad Católica de Valparaíso (PUCV), en Chile. Sus intereses de investigación incluyen: administración educacional; colaboración e internacionalización de la investigación en educación; perspectiva de género; inclusión educacional; trayectorias y transiciones estudiantiles; política educacional y el valor público de la educación. ORCID ID: 0000-0002-7324-9275. Email: juan.queupil@pucv.cl 


\section{Francisco Durán del Fierro}

Sociólogo y Magíster en Gestión y Políticas Públicas. Es profesor en la carrera sociología Universidad de Chile y coordinador e investigador de la Unidad de Análisis y Desarrollo del Departamento de Evaluación, Medición y Registro Educacional (DEMRE). Fue Vicerrector de Planificación y Aseguramiento de la Calidad en la Universidad de Aysén (2016) y coordinador de área de la reforma a la educación superior en el Ministerio de Educación (2014-2016). ORCID ID: O000-0003-0534-5342. Email: fdurandelfierro@u.uchile.cl 